\title{
Overview of Various Measurement Tools for Shoulder Kinematics
}

\author{
Doo Sup Kim ${ }^{\bowtie}$, Myoung Gi On, Jun Seop Yeom \\ Department of Orthopedic Surgery, Wonju Severance Christian Hospital, Yonsei University Wonju College of Medicine, Wonju, Korea
}

\begin{abstract}
Shoulder kinematics is important, as it is associated with shoulder arthropathy and pain mechanisms. Various static and dynamic analysis methods are prevalent for shoulder kinematics. These include 2-dimensional plane x-ray, computed tomography, and magnetic resonance imaging, cadaver study, electromagnetic motion analysis, transcortical bone pins technique, and in vivo 3-dimensional motion analysis. Although these methods provide the value of the shoulder kinematics angle, they are unable to explain why such changes occur. Since each method has its pros and cons, it is important to understand all factors accurately, and to choose a method that best meets the purpose of the researcher.
\end{abstract}

(Clin Shoulder Elbow 2017;20(4):244-249)

Key Words: Shoulder; Kinematics

\section{Introduction}

The shoulder, consisting of three bones (clavicle, humerus, and scapula), is a very complex joint encompassing four joints (glenohumeral joint, scapulothoracic joint, acromioclavicular joint, and sternoclavicular joint), numerous ligaments, and muscles. The shoulder joints maintain very efficient movements and stability by moving these components cooperatively. A problem in any one of these movements affects the other joints, and eventually the patient complains of pain." In addition, the mechanism of the rotator cuff tear (RCT) and pain induction has not been elucidated yet, but many studies suggest that one of the factors is a kinematic change of the shoulder. ${ }^{2-4)}$ In the subacromial impingement syndrome, changes in the glenohumeral joint kinematics, such as the excessive superior translation of the humeral head, have been identified as the cause of pain. ${ }^{5,6)}$ Scapular and humerus are known to move at a constant rate during the elevation of the shoulder, and the scapulohumeral rhythm (SHR) has been elucidated to be in a 2:1 ratio by Inman and Saunders. ${ }^{7)}$ However, recent studies have shown that individual differences are significant, and some degree of difference exists between dominant and non-dominant arms. ${ }^{8)}$ Various diseases around the shoulder are especially known to change the SHR; hence, measuring the SHR can help predict the morbidity of a disease. ${ }^{6,9)}$ Earlier studies mostly used plain radiographs as a means to measure scapular kinematics (upward rotation angle, posterior tilting angle, and external rotation angle) including SHR. ${ }^{10)}$ Plain radiographs. Although still used frequently, there is a limitation to measuring the 3-dimensional (3D) changes of the scapula. Hence, this study evaluates the advantages and disadvantages of three methods, mainly focusing on methods that are frequently used, and with recently applied examples.

\section{Techniques}

\section{Two-dimensional (2D) Plane x-ray}

Since the time it was employed by Inman and Saunders, ${ }^{7)}$ 2D plane $\mathrm{x}$-ray is one of the oldest research methods used for the study of static kinematics. ${ }^{10)}$ Paletta et al. ${ }^{3)}$ compared the kinematics of shoulder abduction in normal, anterior shoulder instability (ASI) and RCT patients using scapular plane and horizontal (axillary) plane $x$-ray. They observed that $39 \%$ of preoperative

Received November 1, 2017. Revised November 20, 2017. Accepted November 21, 2017.

Correspondence to: Doo Sup Kim

Department of Orthopedic Surgery, Wonju Severance Christian Hospital, Yonsei University Wonju College of Medicine, 20 Ilsan-ro, Wonju 26426, Korea

Tel: +82-33-741-0114, Fax: +82-33-736-7326, E-mail: dskim1974@yonsei.ac.kr

Review article does not need an IRB approval.

Financial support: None. Conflict of interests: None. 
ASI patients and $100 \%$ of RCT patients had altered glenohumeral kinematics such as superior translation of humeral head, but normal glenohumeral kinematics were reported in 100\% of the ASI patients and $86 \%$ of RCT patients at 2 years after surgery. Various studies undertaken by Yamaguchi et al. ${ }^{4)}$ included serial $\mathrm{x}$-ray of abduction in normal, symptomatic and asymptomatic RCT patients, and translation of the humeral head. They reported a superior translation of the humeral head during arm abduction in RCT patients.

Although these studies can be performed relatively easily compared with other methods, a limitation of this technique is that a dynamic study is not possible, and the difference in the interobserver and intraobserver reliability are large.

\section{Computed Tomography and Magnetic Resonance Imaging}

Commonly used in medical practice, both computed tomography $(\mathrm{CT})$ and magnetic resonance imaging (MRI) provide useful information regarding the bones and muscles, respectively. Especially, 3D imaging provides a scope beyond the limit of the existing 2D plane, thereby enabling 3D analysis. ${ }^{11,12)}$ It is difficult to accurately measure the critical shoulder angle $(\mathrm{CSA})^{13}$ in $2 \mathrm{D}$ plane kinematics using conventional plain $\mathrm{x}$-rays because of the complicated anatomical structure of the shoulder, such as the scapula. Although a 3D technique enabled the study of the CSA (Fig. 1), the disadvantage of providing only static kinematics at certain angles other than dynamic kinematics still remains valid. Also, it is difficult to capture motion, other than the neutral position, in CT and MRI due to the unique helical and closed structure. Several methods have been studied to overcome

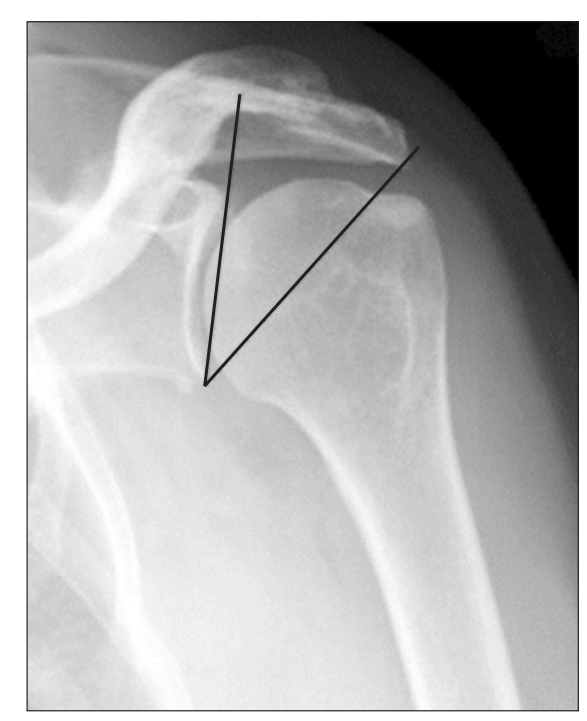

Fig. 1. Assessment of the critical shoulder angle on standardized true anteroposterior radiographs. The angle is formed by a line connecting the superior and inferior border of the glenoid fossa, and a second line connecting the latter with the most infero-lateral point of the acromion. these limitations. Among them, open MRI can be accomplished in a more free posture than the conventional closed MRI, and it is possible to conduct 3D glenohumeral motion for in vivo study. Graichen et al. ${ }^{14)}$ studied the glenohumeral kinematics and muscle activity by performing open MRI at $30^{\circ}$ intervals, with passive and active shoulder abduction at $30^{\circ}$ to $150^{\circ}$. Kim et al. ${ }^{15)}$ studied the acromioclavicular joint motion by performing 3D CT in the neutral position and full abduction, in patients who underwent hook plate fixation with comminuted distal clavicle fracture. They reported that during abduction, the distal clavicle on the side of the hook plate fixation had less internal rotation and more translation of the medial acromion compared to the other side. In addition, Kim et al. ${ }^{16)}$ also studied the relationship between scapular fracture, clavicular fracture and scapular dyskinesis by performing 3D CT in the neutral position and full elevated position. They reported that clavicular fracture had no significant effect on scapular kinematics, but scapular fracture was associated with a decrease in scapular elevation and an increase in the anterior tilt, resulting in the scapular fracture being a cause for scapular dyskinesis.

These studies also performed motion analysis, which is a limitation of existing CT and MRI studies. However, the impossibility of dynamic motion analysis still remains a limitation in this technique.

\section{Cadaver Study}

Debski et al. ${ }^{17)}$ conducted a dynamic study of the glenohumeral joint using six computer-controlled hydraulic cylinders, and Su et al. ${ }^{18)}$ studied the relationship between RCT and glenohumeral translation through a cadaver study using customized zig. The cadaver study allows for dynamic study of joint motion or position, imaging study and direct observation. ${ }^{19)}$

It is a very useful tool for measuring the approximate changing trend of the shoulder kinematic before in vivo measurement since it is possible to study only one limited movement; also, kinematics cannot be studied with the muscles and ligaments of the shoulder being excluded, and this is a controlled test rather than actual physiologic loading. However, there is a limitation for complete reproduction in vivo. Recently, the authors studied shoulder kinematics utilizing cadaveric study with the Vicon motion analysis system (Vicon Motion System, Oxford, UK) with 6 cameras (X-T10 2 megapixel camera; Fujifilm, Bangkok, Thailand) (Fig. 2).

\section{Electromagnetic Motion Analysis}

Electromagnetic motion analysis enables the dynamic analysis of shoulder motion, unlike other modalities such as simple $x$ ray, CT, and MRI. This provides dynamic kinematics information as an electromagnetic tracking method that attaches markers directly to the patient's skin. Kolk et al. ${ }^{20)}$ studied the preoperative and postoperative 1 year shoulder kinematics and shoulder 

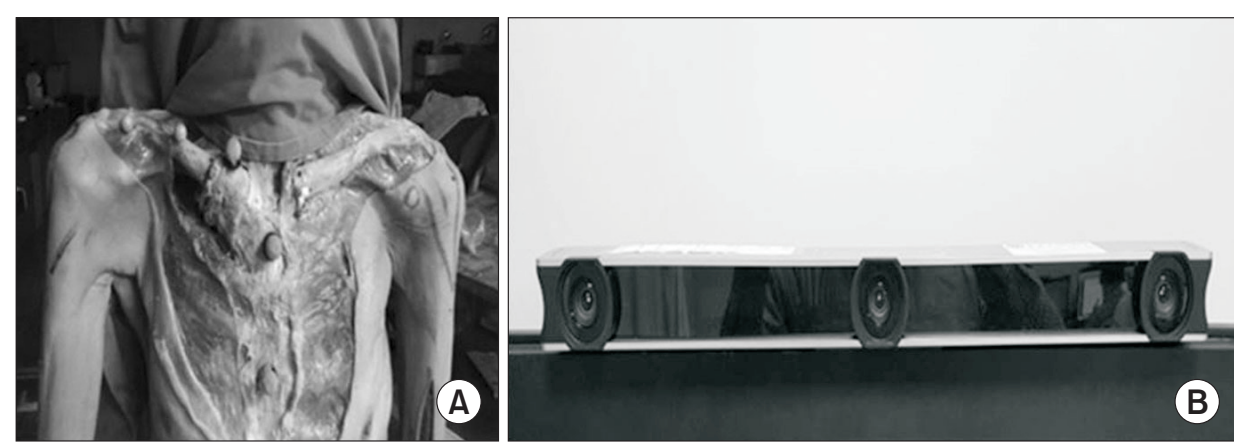

Fig. 2. Five reflective bony markers were attached to the proximal and distal sternum and clavicle to recognize the axis. Vicon motion analysis system (Vicon Motion System, UK) with 6 cameras (MX-T10 2 megapixel camera). range of motion (ROM) in RCT patients using an electromagnetic tracking device. They reported the recovery of shoulder kinematics and an increase in ROM at the postoperative 1-year follow-up. Dayanidhi et al. ${ }^{21)}$ studied scapular kinematics in arm elevation in adults and children using a magnetic tracking device wherein they reported a significant difference between children and adults in scapular kinematics. The greater upward rotation of the scapular and the glenohumeral to scapulothoracic ratio in shoulder abduction was 2.4:1 in adults and 1.3:1 in children. This method can be applied to dynamic studies, such as forward elevation and external rotation, by attaching an electromagnetic sensor to the anatomical landmark of the scapula, humerus, and thoracic. It is also possible to study scapulothoracic motion with glenohumeral motion including the trunk.

However, the electromagnetic tracking method has limitations in that it is difficult to measure the scapular movement for the overhead activity of $120^{\circ}$ or more due to the skin-bone displacement and the error by the marker being located in the skin. ${ }^{20,22)}$ In particular, an error occurs as the elevation angle increases. $^{13,21,23)}$

\section{Transcortical Bone Pins Technique}

McClure et al. ${ }^{24)}$ studied scapular kinematics by inserting pins directly into the subject's bone for direct 3D measurement of scapular kinematics. Karduna et al. ${ }^{25)}$ performed a transcortical bone-pin technique to attach the sensor directly to the bone to remove the skin motion artifact that occurs when the sensor is placed in the skin, as in the previous electromagnetic motion study. Since the sensor pin is placed on the bone such as the scapula, accurate kinematics can be measured; furthermore, flexion motion of $120^{\circ}$ or more can be measured, which is otherwise limited by the existing electromyography (EMG). However, the pin pain may affect kinematics. Also, the invasive study makes it difficult to collect a large number of subjects, and it is difficult to perform repeated examinations before and after surgery.

\section{In Vivo 3D Motion Analysis}

The recently developed shape matching technique using the 3D-to-2D model registration technique was originally used for in vivo 3D motion analysis of total knee arthroplasty. ${ }^{26)}$ However, this has now been extended to the spine, hip and shoulder in vivo measurements. ${ }^{27-29)}$ Recently, a bi-plane fluoroscopy method was used to reduce errors in out of plane. ${ }^{30)}$ Shape matching technique has the disadvantage of radiation hazard, and it is difficult to measure the relationship with other joints since the measurement site is confined to one joint only. However, more accurate dynamic kinematic information can be obtained as compared to other methods, and the accessibility of researchers can be improved by using fluoroscopy, which is commonly available in hospitals. Although the shape matching technique is a very accurate method, it is also an indirect tool. Shape matching technique has recently become popular because it provides a kinematic value that reflects the in vivo state, and can be linked to the clinic. Since the preoperative and postoperative clinical results are correlated with the kinematic value, there are high expectations that they will be able to measure and compensate for the deficient part in cooperation with the muscles.

\section{1) Shape matching technique based on single plane fluoroscopy}

This is a suitable method to measure the dynamic kinematics of the glenohumeral joint, such as the relationship between posterior tilt, external rotation, upward rotation of the scapula and SHR, relationship between superior-inferior translation, internalexternal rotation and scapular value, and relationship between scapula and humerus. ${ }^{8,27)}$ This is achieved by matching the 3D image acquired by the CT to the dynamic 2D image obtained by using fluoroscopy. ${ }^{28,29)}$ Recently, we used a shape matching technique to study the effects of short malunion of the clavicle on in vivo scapular kinematics (Fig. 3, 4). ${ }^{31)}$ Our results showed that clavicle shortening of $>10 \%$ greatly affects the scapular kinematics in vivo. The change in the length of clavicle (one of the bones constituting the shoulder joint) eventually leads to a change in scapular kinematics. Matsumura et al. ${ }^{32)}$ conducted a cadaver study on scapular kinematics in studying the shortening deformities of the clavicles with almost the same subject. Although both study results were almost similar, the in vivo studies, external rotation and posterior tilting of the scapula increased significantly from $100^{\circ}$ to maximum abduction, but did not show any difference according to the elevation angle in the cadaver study. This 

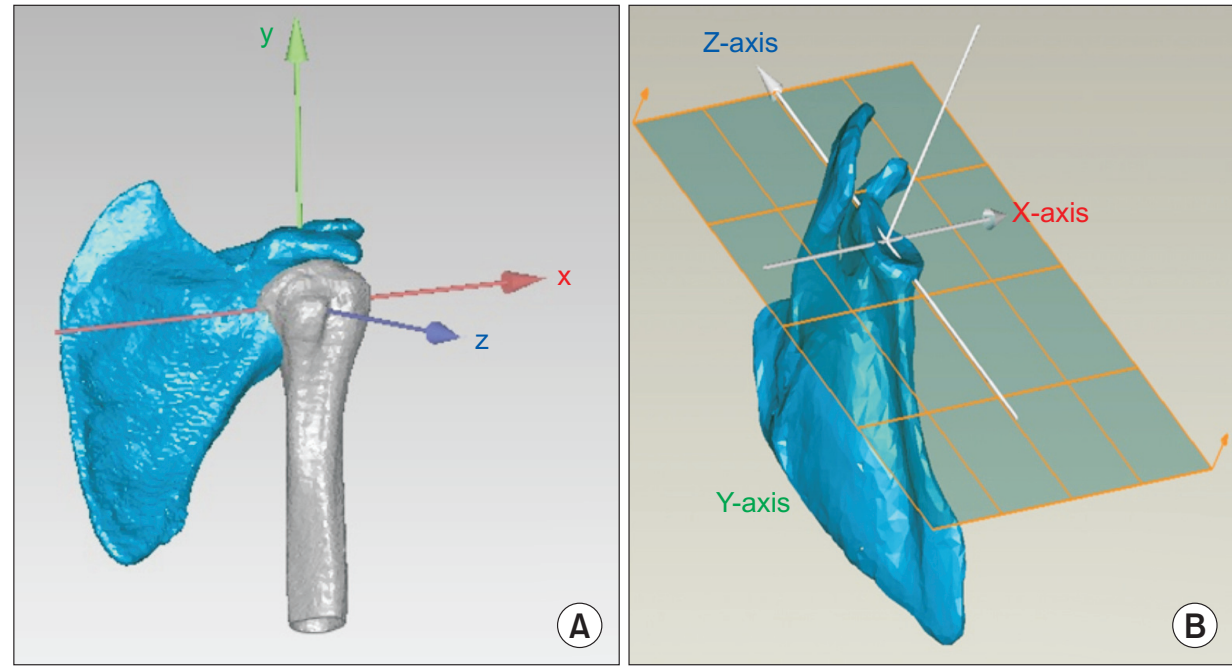

Fig. 3. Using computed tomography images, 3-dimensional (3D) models of the humerus, scapula, and clavicle were constructed with ITKSNAP (Penn Image Computing and Science Laboratory, Philadelphia, PA, USA), and anatomic coordination was assigned to $3 \mathrm{D}$ models of the humerus $(\mathrm{A})$ and glenoid (B) with Geomagic studio (X-axis, centroid of the humeral head; Y-axis, parallel to the humeral shaft; Z-axis, intertubercular groove).
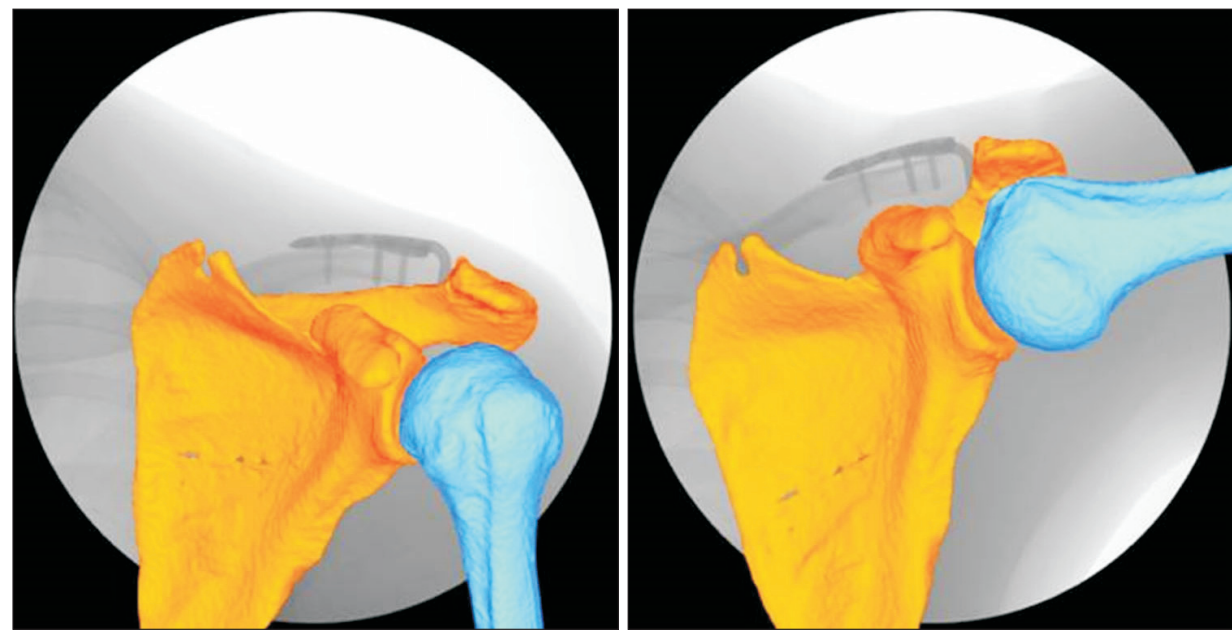

Fig. 4. Three-dimensional (3D) to 2-dimensional model-to-image registration was used to determine 3D motion of the scapula and humerus during dynamic arm elevation.

reflects the difference between in vivo studies (which reflect realtime muscle conditions) and the cadaver studies.

The limitations of the shape matching technique are that the risk of ionizing radiation is higher than other methods, and accuracy is less than that of the external plane change due to the use of single plane fluoroscopy. Also, the study includes the thorax, unlike the electromagnetic motion analysis.

\section{2) Radiostereometric analysis technique based on dual} plane fluoroscopy

The shape matching technique using single plane fluoroscopy has the drawback that it is difficult to detect out of plane translation as compared with in plane translation. On the other hand, dual plane fluoroscopy is emerging as an alternative. Bey et al. ${ }^{30,33)}$ analyzed the dynamic 2D image obtained by biplane $\mathrm{x}$-ray system, and 3D scapula and humerus bone model obtained by bilateral CT using model-based tracking technique. This method provides six kinematic parameters (three rotations, three translations), which can be used to obtain a more accurate understanding of the changes in the humerus motion as the scapula changes, as compared to EMG. In addition, it is possible to study the joint contact pattern by measuring the minimum distance between the glenoid and the humerus in each $2 \mathrm{D} \mathrm{im-}$ age. $^{34)}$

However, it has a disadvantage that since this is also a CT based bone model, the cartilage is excluded from the joint contact pattern, and also radiation hazard using biplane x-ray and $\mathrm{CT}$ is higher than in other studies.

\section{Conclusion}

There are static and dynamic analytical methods for shoulder kinematics. All methods described above provide valuable shoulder kinematics angle, although they are unable to explain why such changes occur. Although EMG should be performed simultaneously to measure muscle changes around the shoulder joint, there are many technical difficulties in clinical studies. In fact, even if EMG is performed, it is necessary to solve the problem of measuring the muscle state of the moment that causes 
real-time kinematics change, namely, the problem of synchronization. Hence, more researches need to be conducted in this area. The methods introduced earlier each have advantages and disadvantages. It is important to understand this correctly and choose a measurement method that meets the purpose of the researcher.

\section{References}

1. Cutti AG, Veeger HE. Shoulder biomechanics: today's consensus and tomorrow's perspectives. Med Biol Eng Comput. 2009;47(5):463-6.

2. Keener JD, Wei AS, Kim HM, Steger-May K, Yamaguchi K. Proximal humeral migration in shoulders with symptomatic and asymptomatic rotator cuff tears. J Bone Joint Surg Am. 2009;91(6):1405-13.

3. Paletta GA Jr, Warner JJ, Warren RF, Deutsch A, Altchek DW. Shoulder kinematics with two-plane x-ray evaluation in patients with anterior instability or rotator cuff tearing. J Shoulder Elbow Surg. 1997;6(6):516-27.

4. Yamaguchi K, Sher JS, Andersen WK, et al. Glenohumeral motion in patients with rotator cuff tears: a comparison of asymptomatic and symptomatic shoulders. J Shoulder Elbow Surg. 2000;9(1):6-11.

5. Poppen NK, Walker PS. Normal and abnormal motion of the shoulder. J Bone Joint Surg Am. 1976;58(2):195-201.

6. Warner JJ, Micheli LJ, Arslanian LE, Kennedy J, Kennedy R. Scapulothoracic motion in normal shoulders and shoulders with glenohumeral instability and impingement syndrome. A study using Moiré topographic analysis. Clin Orthop Relat Res. 1992;(285):191-9.

7. Inman VT, Saunders JB. Observations on the function of the clavicle. Calif Med. 1945;65(4):158-66.

8. Matsuki K, Matsuki KO, Mu S, et al. In vivo 3-dimensional analysis of scapular kinematics: comparison of dominant and nondominant shoulders. J Shoulder Elbow Surg. 2011; 20(4):659-65.

9. Uhl TL, Kibler WB, Gecewich B, Tripp BL. Evaluation of clinical assessment methods for scapular dyskinesis. Arthroscopy. 2009;25(11):1240-8.

10. Freedman L, Munro RR. Abduction of the arm in the scapular plane: scapular and glenohumeral movements. A roentgenographic study. J Bone Joint Surg Am. 1966;48(8):1503-10.

11. Baeyens JP, Van Roy P, De Schepper A, Declercq G, Clarijs JP. Glenohumeral joint kinematics related to minor anterior instability of the shoulder at the end of the late preparatory phase of throwing. Clin Biomech (Bristol, Avon). 2001;16(9):752-7.

12. von Eisenhart-Rothe RM, Jäger A, Englmeier $\mathrm{KH}$, Vogl TJ, Graichen $\mathrm{H}$. Relevance of arm position and muscle activity on three-dimensional glenohumeral translation in patients with traumatic and atraumatic shoulder instability. Am J Sports
Med. 2002;30(4):514-22

13. Moor BK, Bouaicha S, Rothenfluh DA, Sukthankar A, Gerber C. Is there an association between the individual anatomy of the scapula and the development of rotator cuff tears or osteoarthritis of the glenohumeral joint?: a radiological study of the critical shoulder angle. Bone Joint J. 2013;95(7):935-41.

14. Graichen $\mathrm{H}$, Stammberger $\mathrm{T}$, Bonel $\mathrm{H}$, Englmeier $\mathrm{KH}$, Reiser $\mathrm{M}$, Eckstein F. Glenohumeral translation during active and passive elevation of the shoulder: a 3D open-MRI study. J Biomech. 2000;33(5):609-13.

15. Kim YS, Yoo YS, Jang SW, Nair AV, Jin $H$, Song HS. In vivo analysis of acromioclavicular joint motion after hook plate fixation using three-dimensional computed tomography. J Shoulder Elbow Surg. 2015;24(7):1106-11.

16. Kim E, Park JH, Han BR, et al. In vivo analysis of three-dimensional dynamic scapular dyskinesis in scapular or clavicular fractures. Acta Med Okayama. 2017;71(2):151-9.

17. Debski RE, McMahon PJ, Thompson WO, Woo SL, Warner JJ, Fu FH. A new dynamic testing apparatus to study glenohumeral joint motion. J Biomech. 1995;28(7):869-74.

18. Su WR, Budoff JE, Luo ZP. The effect of posterosuperior rotator cuff tears and biceps loading on glenohumeral translation. Arthroscopy. 2010;26(5):578-86.

19. Payne LZ, Deng XH, Craig EV, Torzilli PA, Warren RF. The combined dynamic and static contributions to subacromial impingement. A biomechanical analysis. Am J Sports Med. 1997; 25(6):801-8.

20. Kolk A, de Witte PB, Henseler JF, et al. Three-dimensional shoulder kinematics normalize after rotator cuff repair. J Shoulder Elbow Surg. 2016;25(6):881-9.

21. Dayanidhi S, Orlin M, Kozin S, Duff S, Karduna A. Scapular kinematics during humeral elevation in adults and children. Clin Biomech (Bristol, Avon). 2005;20(6):600-6.

22. Kai Y, Gotoh M, Takei K, et al. Analysis of scapular kinematics during active and passive arm elevation. J Phys Ther Sci. 2016;28(6):1876-82.

23. Fayad F, Roby-Brami A, Yazbeck C, et al. Three-dimensional scapular kinematics and scapulohumeral rhythm in patients with glenohumeral osteoarthritis or frozen shoulder. J Biomech. 2008;41(2):326-32.

24. McClure PW, Michener LA, Sennett BJ, Karduna AR. Direct 3-dimensional measurement of scapular kinematics during dynamic movements in vivo. J Shoulder Elbow Surg. 2001; 10(3):269-77.

25. Karduna AR, McClure PW, Michener LA, Sennett B. Dynamic measurements of three-dimensional scapular kinematics: a validation study. J Biomech Eng. 2001;123(2):184-90.

26. Banks SA, Hodge WA. Accurate measurement of threedimensional knee replacement kinematics using single-plane fluoroscopy. IEEE Trans Biomed Eng. 1996;43(6):638-49.

27. Kijima T, Matsuki K, Ochiai N, et al. In vivo 3-dimensional 
analysis of scapular and glenohumeral kinematics: comparison of symptomatic or asymptomatic shoulders with rotator cuff tears and healthy shoulders. J Shoulder Elbow Surg. 2015; 24(11):1817-26.

28. Matsuki K, Matsuki KO, Yamaguchi S, et al. Dynamic in vivo glenohumeral kinematics during scapular plane abduction in healthy shoulders. J Orthop Sports Phys Ther. 2012;42(2):96104.

29. Nishinaka N, Tsutsui H, Mihara K, et al. Determination of in vivo glenohumeral translation using fluoroscopy and shapematching techniques. J Shoulder Elbow Surg. 2008;17(2):31922.

30. Bey MJ, Kline SK, Zauel R, Lock TR, Kolowich PA. Measuring dynamic in-vivo glenohumeral joint kinematics: technique and preliminary results. J Biomech. 2008;41(3):711-4.
31. Kim D, Lee D, Jang Y, Yeom J, Banks SA. Effects of short malunion of the clavicle on in vivo scapular kinematics. J Shoulder Elbow Surg. 2017;26(9):e286-92.

32. Matsumura N, Ikegami H, Nakamichi N, et al. Effect of shortening deformity of the clavicle on scapular kinematics: a cadaveric study. Am J Sports Med. 2010;38(5):1000-6.

33. Bey MJ, Zauel R, Brock SK, Tashman S. Validation of a new model-based tracking technique for measuring three-dimensional, in vivo glenohumeral joint kinematics. J Biomech Eng. 2006;128(4):604-9.

34. Bey MJ, Peltz CD, Ciarelli K, et al. In vivo shoulder function after surgical repair of a torn rotator cuff: glenohumeral joint mechanics, shoulder strength, clinical outcomes, and their interaction. Am J Sports Med. 2011;39(10):2117-29. 